\title{
Improved Filtering of Pulse Oximeter Monitoring Alarms in the Neonatal ICU: Bedside Significance
}

\author{
Beatrice M Stefanescu MD MSc, T Michael O’Shea MD MPH, Fran Haury RRT, \\ Waldemar A Carlo MD, and James C Slaughter PhD
}

\begin{abstract}
BACKGROUND: The OxiMax N-600x containing SatSeconds alarm management software was designed to assist clinicians in discriminating nuisance alarms from those that are clinically relevant. Instead of sounding an alarm the moment the oxygen saturation reading violates the upper or lower limit settings, a magnitude and duration of tolerance can be set. The primary objective was to study the proportion of nuisance alarms relative to the proportion of clinically relevant alarms being filtered under 4 different SatSeconds alarm settings (ie, 10, 25, 50, and 100) in the neonatal intensive care environment. METHODS: This is an observational prospective study of 50 infants cared for in 3 large neonatal ICUs. Infants were monitored for 4 continuous $h$ each by a study observer with the study monitor, in addition to standard multiparameter monitors. The performance of the SatSeconds alarm was compared with nursing intervention. RESULTS: The area under the receiver operating characteristic curve $(95 \%$ CI ) for SatSeconds alarm settings when compared with nursing intervention were as follows: $0.61(0.57-0.66)$ when the SatSeconds alarm setting was off, $0.63(0.59-0.68)$ for the 10 SatSeconds alarm setting, $0.64(0.59-0.69)$ for the 25 SatSeconds alarm setting, $0.64(0.59-0.69)$ for the 50 SatSeconds alarm setting, and $0.63(0.58-$ 0.68) for the 100 SatSeconds alarm setting, respectively. CONCLUSIONS: The SatSeconds feature of the OxiMax N-600x pulse oximeter reduced some nuisance alarms; however, its specificity to nurse-identified desaturation events does not significantly improve with lengthening SatSeconds alarm settings. Key words: nuisance alarms; $S_{p_{2}}$; Nellcor OxiMax N-600x; SatSeconds alarm settings. [Respir Care 2016;61(1):85-89. (C) 2016 Daedalus Enterprises]
\end{abstract}

\section{Introduction}

Recognizing the potential safety issues that the medical device alarms may pose, on April 8, 2013, the Joint Commission released a Sentinel Event Alert with recommendations to healthcare organizations to reduce patient harm related to medical device alarm systems. ${ }^{1}$ The performance

\footnotetext{
Dr Stefanescu is affiliated with the Department of Pediatrics, Vanderbilt University School of Medicine, Nashville, Tennessee. Dr O'Shea is affiliated with the Department of Pediatrics, Wake Forest School of Medicine, Winston-Salem, North Carolina. Ms Haury is affiliated with Clinical Affairs, Respiratory and Monitoring Solutions, Covidien, Boulder, Colorado. Dr Carlo is affiliated with the Department of Pediatrics, University of Alabama at Birmingham, Birmingham, Alabama. Dr Slaughter is affiliated with the Department of Biostatistics, Vanderbilt University School of Medicine, Nashville, Tennessee.
}

This study was supported by Nellcor/Covidien. Ms Haury is an employee of Covidien. The other authors have disclosed no conflicts of interest. of pulse oximeters is of particular importance in the neonatal ICU because of the danger that both hyperoxemia and hypoxemia pose to newborns; hyperoxemia is associated with an increased risk of bronchopulmonary dysplasia or retinopathy of prematurity, and even small differences in oxygen saturation targets influence mortality risk. ${ }^{2-5}$ High false alarm rates contribute to the problem of alarm fatigue and could endanger patients if clinicians become inured to the continual alarms and ignore some that may be clinically relevant. ${ }^{6-8}$ Further, frequent false alarms could lead

\footnotetext{
Correspondence: Beatrice Stefanescu MD MSc, Vanderbilt University School of Medicine, Monroe Carell Jr. Children's Hospital at Vanderbilt, 11116 Doctor's Office Tower, 2200 Children's Way, Nashville, TN 27232 9544. E-mail: beatrice.stefanescu@ Vanderbilt.edu.
}

DOI: $10.4187 /$ respcare. 04177 


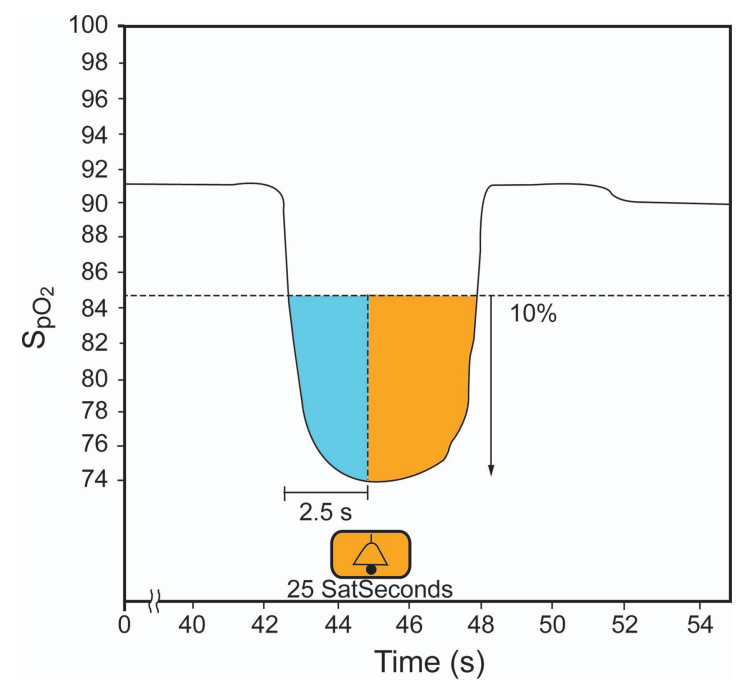

Fig. 1. An example with SatSeconds set at 25. The alarm will sound (orange area) when the subject's monitored pulse oximetry saturation is $10 \%$ below the set lower limit for $2.5 \mathrm{~s}$.

to unnecessary handling of critically ill neonates who often do not tolerate such handling and increased noise. ${ }^{9-12}$

For these reasons, pulse oximeters have been developed to provide a more accurate alarm system; these oximeters rely upon algorithms to filter out the background distortions associated with motion. ${ }^{13-16} \mathrm{~A}$ comparison of a newer-generation monitoring system with older-generation monitors in preterm neonates showed that false-alarm rates varied greatly, with the newer-generation monitoring system having a significantly lower rate. ${ }^{17}$ Forty percent of total false alarms were due to pulse oximeters, and most of the false alarms could be ascribed to motion (eg, signal loss or displaced sensors).

The Nellcor OxiMax N-600x monitoring system contains SatSeconds alarm management software designed to assist clinicians in discriminating nuisance alarms from those that are clinically relevant. Instead of sounding an alarm the moment the $\mathrm{S}_{\mathrm{pO}_{2}}$ reading violates the upper or lower limit settings, a magnitude and duration of tolerance can be set. Clinicians may set one of 4 predefined SatSeconds settings: 10, 25, 50, or 100 SatSeconds. The SatSeconds alarm measures the number of percentage points by which the $\mathrm{S}_{\mathrm{pO}_{2}}$ violates the saturation alarm limit and multiplies this value by the duration of the violation in seconds (Fig. 1). For example, a low alarm setting of 90 and a SatSeconds setting of 10 will trigger an alarm if a saturation reading of 89 occurs for $>10 \mathrm{~s}, 88$ for $>5 \mathrm{~s}, 85$ for $>2 \mathrm{~s}$, or any combination of magnitude and time that produces 10. As a safety feature, if $\geq 3 \mathrm{~S}_{\mathrm{pO}_{2}}$ violations occur within a 60 -s period, an alarm will sound. Thus, the software will not sound an alarm every time $\mathrm{S}_{\mathrm{pO}_{2}}$ levels drop below the target limit, filtering out some alarms. The purpose of this study was to evaluate the proportion of

\section{QUICK LOOK}

\section{Current knowledge}

The use of pulse oximetry in the neonatal ICU is a standard of care to prevent both hyperoxemia and hypoxemia. Frequent nuisance alarms related to motion, probe displacement, and low perfusion can obfuscate alarm management due to alarm fatigue. Alarm management, including the use of smart alarms, has been developed to reduce nuisance alarms and improve patient safety.

\section{What this paper contributes to our knowledge}

In an observational study of 50 neonates, a new alarm algorithm was compared with clinical monitors. Caregivers were blinded to the research oximeter. The new algorithm slightly improved filtering of transient and non-clinically meaningful interventions; however, it did not maintain a high specificity to nurse-identified desaturation events with lengthening of the alarm setting from 10 to $100 \mathrm{~s}$.

nuisance alarms relative to the proportion of clinically relevant alarms being filtered under 4 different SatSeconds settings (ie, 10, 25, 50, and 100) in the neonatal ICU setting.

\section{Methods}

We conducted an observational prospective study of 50 infants in the neonatal ICU at 3 hospitals in the United States: MetroHealth Medical Center in Cleveland, Ohio (Center A), University of Alabama at Birmingham in Birmingham, Alabama (Center B), and Wake Forest Baptist Health in Winston-Salem, North Carolina (Center C). All infants in the neonatal ICU were eligible for the study as long as they were receiving continuous $\mathrm{S}_{\mathrm{pO}_{2}}$ monitoring for their routine care. Infants whose parents or legal guardians did not provide consent to the study or who did not understand and read English were not eligible to participate in this study.

Participating infants were attached to the Nellcor OxiMax N-600x pulse oximeter with its disposable sensor, in addition to standard multiparameter monitors. The blinded study pulse oximeter was attached to all participants to capture oxygen saturation data with its alarm muted. The standard multiparameter monitors were used by clinical staff for the evaluation and treatment of patients. Upper and lower oxygen saturation alarm limit settings to the standard care oximeter were determined for each infant individually by the neonatal ICU staff following the stan- 


\section{Pulse Oximeter Monitoring Alarms in the NICU}

dard of care in each participating neonatal ICU. There were 4 standard function pulse oximeters that were used in the study: Phillips Intellivue MP70 (Philips Healthcare, Andover, Massachusetts) with Masimo technology (Masimo, Irvine, California), GE Solar 8000i (GE Healthcare, Madison, Wisconsin) with Nellcor N-600x technology (Covidien llc, Mansfield, Massachusetts), Intellivue module (Philips Healthcare) with Masimo SET technology (Masimo), and Phillips Medizin System M3001-A (Philips Healthcare) with Nellcor technology (Covidien llc).

The neonatal ICU staff responsible for the care of study subjects was observed by a study observer for $4 \mathrm{~h}$ continuously for each infant. The study observer was an employee of Pharmaceutical Product Development, an independent contract research organization. This observer underwent comprehensive training to ensure consistency in the definition and detection of clinical interventions and to minimize intrusion. The items that were noted by the observer during the observation period included clinical interventions by neonatal ICU staff and time of intervention, time of alarm occurrence, $\mathrm{S}_{\mathrm{pO}_{2}}$ target limits for each infant, and sensor site (eg, foot or hand). The precipitating event to elicit nursing interventions was oxygen desaturation alarms on the standard monitor. Observed clinical interventions included position change, tactile stimulation, lung auscultation, supplemental oxygen adjustment, ventilator setting changes, upper and/or lower airway suctioning, administration of positive-pressure ventilation, and administration of cardio-respiratory resuscitation. These data were collected on an intervention checklist. The time of each event was also taken from a download of the oximeters, with a level of accuracy to the second. Because infant characteristics may affect the frequency of alarms, select infant data were collected, including chronological age, gestational age at birth, sex, weight at birth and current weight, and ethnicity. The author associated with Covidien did not have access to the contract research organization observer.

The primary objective of this study was to determine the proportion of alarms with associated clinically relevant events, defined as an alarm leading to an observed clinical intervention within $3 \mathrm{~min}$. The analyses were performed for each SatSeconds setting $\left(10,25,50\right.$, and 100). $\mathrm{S}_{\mathrm{pO}_{2}}$ data were analyzed to identify the occurrence of alarms at each of the SatSeconds settings. The occurrence and timing of the alarms from SatSeconds were matched to the occurrence and timing of clinical interventions as determined by the observer.

A sample of 50 infants in the neonatal ICU provided adequate precision to estimate the mean proportion of alarms associated with clinically relevant events among all infants to within 0.28 SD with $95 \%$ confidence. We computed the frequency and proportion of alarms with corresponding 95\% CIs with associated clinically relevant events at each of the 4 SatSeconds settings. Descriptive analyses of baseline infant information were conducted using frequencies and other univariate statistics. Differences in alarm frequencies and proportions were compared and tested across levels of baseline infant data using the chi-square test of significance. A true positive event was defined as an alarm that preceded a clinical intervention by the infant's nurse; a false negative event was defined as a clinical intervention by the nurse that was not preceded by a study monitor alarm; a false positive event was defined as an alarm that was not followed by a clinical intervention by the nurse; and a true negative event was defined as an alarm on the standard care oximeter that was not followed by a clinical intervention by the nurse and was not accompanied by an alarm of the study oximeter. A receiver operator curve analysis was performed for each SatSeconds parameter studied $(0,10,25,50$, and 100 , respectively) to define its individual predictive value for the need for a clinically meaningful intervention. All confidence intervals were based on a $5 \%$ significance level.

\section{Ethics}

Approval for the study was obtained from each participating hospital institutional review board. The research was conducted in accordance with the ethical standards of all applicable national and institutional committees and the World Medical Association's Helsinki Declaration. Individual consent to study participation was obtained from each infant's parent.

\section{Results}

Informed consent was obtained for voluntary participations for 55 subjects at 3 participating nurseries: 23 subjects at Center A, 16 subjects at Center B, and 16 subjects at Center C. Four infants at Center A were discharged from the neonatal ICU before being able to participate. One parent withdrew consent at Center C. Fifty infants completed the study. All subjects were monitored for 4 continuous h with the test oximeter. Demographic characteristics included infants with gestational age ranging from 23 to 40 weeks (mean 32, SD 5), weight at the time of the study ranged from 774 to $6,725 \mathrm{~g}$ (mean 2,726, SD 1,317), and age at the time of the study ranged from $1 \mathrm{~d}$ to 36 weeks (mean 6.6 weeks, SD 8.8). There were 21 (42\%) female infants included in the study. Thirty-two (64\%) of the study participants were white, 17 (34\%) were black, and $1(2 \%)$ was Asian.

Both standard and study pulse oximeters were attached to a lower extremity in 23 infants. The standard pulse oximeter was attached to a lower extremity, whereas the study pulse oximeter was attached to an upper extremity in 26 infants. In the latter case, the presence of a peripheral 


\section{Pulse Oximeter Monitoring Alarms in the NICU}

Table 1. Sensitivity, Specificity, Positive Predictive Value, Negative Predictive Value, Area Under the Curve, and 95\% CIs for the 0, 10, 25, 50, and 100 SatSeconds Settings When Compared With Nursing Intervention

\begin{tabular}{|c|c|c|c|c|c|}
\hline Test Variable & Sensitivity & Specificity & PPV & NPV & $\operatorname{AUC}(95 \% \mathrm{CI})$ \\
\hline SatSeconds alarm off & 0.84 & 0.389 & 0.106 & 0.965 & $0.61(0.57-0.66)$ \\
\hline 10 SatSeconds alarm & 0.81 & 0.46 & 0.114 & 0.965 & $0.63(0.59-0.68)$ \\
\hline 25 SatSeconds alarm & 0.78 & 0.50 & 0.120 & 0.964 & $0.64(0.59-0.69)$ \\
\hline 50 SatSeconds alarm & 0.74 & 0.54 & 0.121 & 0.959 & $0.64(0.59-0.69)$ \\
\hline 100 SatSeconds alarm & 0.7 & 0.57 & 0.123 & 0.956 & $0.63(0.58-0.68)$ \\
\hline $\begin{array}{l}\mathrm{PPV}=\text { positive predictive valu } \\
\text { NPV }=\text { negative predictive val } \\
\text { AUC }=\text { area under the curve }\end{array}$ & & & & & \\
\hline
\end{tabular}

intravenous line precluded the attachment of the study pulse oximeters to the opposite extremity. In one case, both the standard and the study pulse oximeters were attached to the upper extremity. None of the standard care pulse oximeters had alarm delays built in.

Alarm events were recorded at $0,10,25,50$, and 100 SatSeconds. There were a total of 6,496 incidences of standard threshold-based $\mathrm{S}_{\mathrm{pO}_{2}}$ alarms, both upper and lower limit, during $195 \mathrm{~h}$ of monitoring, giving an average of 1 alarm every $2 \mathrm{~min}$. Of these, 5,670 (87.3\%) alarm events were due to oxygen desaturations. The vast majority of threshold-defined oxygen desaturations were very short (mean $12.7 \mathrm{~s}$, SD 34.6); $50 \%$ were $\leq 5 \mathrm{~s}$, and $75 \%$ were $\leq 10$ s. On average, $1.6 \%$ of the alarms were followed by an intervention (eg, tactile stimulation, infant repositioning, airway suctioning, supplemental oxygen adjustment, ventilator setting changes, use of an Ambu bag to ventilate, and administration of CPR).

SatSeconds reduced the total alarm number by 23, 31, 36 , and $40 \%$ at SatSeconds settings of 10, 25, 50, and 100, respectively. Clinically relevant alarms were alarms that required nursing intervention. Table 1 illustrates the sensitivity, specificity, area under the curve, and $95 \%$ CIs for the $0,10,25,50$, and 100 SatSeconds settings when compared with nursing intervention. Neither positive predictive value nor negative predictive value was significantly affected by SatSeconds individual threshold alarm settings (positive predictive value only minimally increased from 0.106 with the SatSeconds alarm off to 0.123 with the 100 SatSeconds alarm; negative predictive value minimally decreased from 0.965 with the SatSeconds alarm off to 0.956 with the 100 SatSeconds alarm).

\section{Discussion}

The technique for oxygen saturation alarm filtering that is described in this report, which incorporates both the magnitude and the duration of the event, reduced the number of clinically irrelevant alarms in our study. Lengthening the SatSeconds alarm setting decreased nuisance alarms, as described by Brostowicz and Rais-Bahrami, ${ }^{18}$ but only slightly improved the receiver-operator curve. There was no additional benefit of increasing beyond the 25 SatSeconds setting on the receiver-operator curve. The advantage of decreasing false alarms with an increased SatSeconds setting was offset largely by the lower sensitivity.

There are several limitations to our study. We used a convenience sample that probably was not fully representative of neonatal ICU patients. This study does not account for clinical interventions due to false alarms and might have included alarms that resulted from routine standard of care (eg, suctioning). Whereas the Nellcor pulse oximeter captured oxygen saturation data from the infant for the purposes of this study, neonatal ICU staff was not aware when the device's alarm would have sounded, so that they would not have to respond to two oximeters. Thus, some alarms from the study oximeter that were categorized as false alarms (because no response was made by the bedside nurse) might have been misclassified. The effect of this bias would be to decrease the observed specificity. In addition, because $\mathrm{S}_{\mathrm{pO}_{2}}$ data were collected electronically from only the Nellcor pulse oximeters, it was not possible to compare the $\mathrm{S}_{\mathrm{pO}_{2}}$ levels between the standard and the study oximeters.

The site of oxygen saturation measurement can affect the observed oxygen saturation values. In our study, the discriminatory placement (right or left) of the study pulse oximeter probe on the extremity was not recorded. This information would be very important, especially in the first hours/days of life, when the ductus arteriosus may be potentially patent. Only one infant of 36 weeks gestation had the probe placement of both pulse oximeters on the upper extremities in its first week of life, and this should not significantly affect the overall results. In a large observational study, Rüegger et $\mathrm{al}^{19}$ showed that with the exception of situations where ductal dependent congenital heart defects or persistent pulmonary hypertension conditions are present, pulse oximetry saturation on both hands can be considered equally preductal. With one exception, 


\section{Pulse Oximeter Monitoring Alarms in the NICU}

the infants were unaffected by these 2 conditions at the time they were studied.

The SatSeconds feature measures the magnitude and duration of the signal without accounting for the quality of the signal, which is a drawback to this method of filtering. Another potential limitation is the fact that the reduction of some alarm frequencies categorized as nuisance alarms with SatSeconds technology may not be beneficial to the infant because there is evidence that even brief desaturation events may have significant clinical consequences if they occur frequently. ${ }^{5}$ As suggested by the work by Di Fiore et $\mathrm{al},{ }^{20}$ even brief intermittent hypoxemic events in extremely preterm infants may contribute to the development of severe retinopathy of prematurity. In order to avoid using the longer SatSeconds setting, which may mask important frequent short oxygen desaturations, training staff in how to use SatSeconds technology becomes extremely important to enhance the benefit and to avoid harm in neonates monitored with a Nellcor OxiMax N-600x pulse oximeter.

\section{Conclusion}

The SatSeconds feature of the Nellcor OxiMax N-600x pulse oximeter slightly improves filtering of transient and non-clinically meaningful interventions; however, it does not maintain a high specificity to nurse-identified desaturation events with lengthening SatSeconds alarm settings. These findings should inform further research into the optimal modality of managing bedside pulse oximeter alarms.

\section{ACKNOWLEDGMENTS}

We thank the study participants and their parents.

\section{REFERENCES}

1. The Joint Commission. Sentinel Event Alert Issue 50: Medical device alarm safety in hospitals. Apr 8, 2013.

2. Bourbon J, Boucherat O, Chailley-Heu B, Delacourt C. Control mechanisms of lung alveolar development and their disorders in bronchopulmonary dysplasia. Pediatr Res 2005;57(5):38R-46R

3. The STOP-ROP Multicenter Study Group. Supplemental therapeutic oxygen for prethreshold retinopathy of prematurity (STOP-ROP), a randomized, controlled trial. I: primary outcomes. Pediatrics 2000; 105(2):295-310.

4. SUPPORT Study Group of the Eunice Kennedy Shriver NICHD Neonatal Research Network. Target ranges of oxygen saturation in extremely preterm infants. N Engl J Med 2010;362(21):1959-1969.

5. BOOST II United Kingdom Collaborative Group, BOOST II Australia Collaborative Group, BOOST II New Zealand Collaborative Group, Stenson BJ, Tarnow-Mordi WO, Darlow BA, et al. N Engl J Med 2013;368(22):2094-2104.

6. Sendelbach S, Funk M. Alarm fatigue: a patient safety concern. AACN Adv Crit Care 2013;24(4):378-386.

7. Hay WW Jr., Rodden DJ, Collins SM, Melara DL, Hale KA, Fashaw LM. Reliability of conventional and new pulse oximetry in neonatal patients. J Perinatol 2002;22(5):360-366.

8. Salyer JW. Neonatal and pediatric pulse oximetry. Respir Care 2003; 48(4):386-396; discussion 397-398

9. Lawless ST. Crying wolf: false alarms in a pediatric intensive care unit. Crit Care Med 1994;22(6):981-985.

10. Sabar R, Zmora E. Nurses' response to alarms from monitoring systems in NICU. Pediatr Res 1997;41(4):174.

11. Long JG, Lucey JF, Philip AG. Noise and hypoxemia in the intensive care nursery. Pediatrics 1980;65(1):143-145.

12. Long JG, Philip AG, Lucey JF. Excessive handling as a cause of hypoxemia. Pediatrics 1980;65(2):203-207.

13. Bohnhorst B, Peter CS, Poets CF. Pulse oximeters' reliability in detecting hypoxemia and bradycardia: comparison between a conventional and two new generation oximeters. Crit Care Med 2000; 28(5):1565-1568.

14. Laptook AR, Salhab W, Allen J, Saha S, Walsh M. Pulse oximetry in very low birth weight infants: can oxygen saturation be maintained in the desired range? J Perinatol 2006;26(6):337-341.

15. Giuliano KK, Higgins TL. New-generation pulse oximetry in the care of critically ill patients. Am J Crit Care 2005;14(1):26-37.

16. Workie FA, Rais-Bahrami K, Short BL. Clinical use of new generation pulse oximeters in the neonatal intensive care unit. Am J Perinatol 2005;22(7):357-360.

17. Ahlborn V, Bohnhorst B, Peter CS, Poets CF. False alarms in very low birthweight infants: comparison between three intensive care monitoring systems. Acta Paediatr 2000;89(5):571-576.

18. Brostowicz HM, Rais-Bahrami K. Oxygen saturation monitoring in the neonatal intensive care unit: evaluation of a new alarm management. J Neonatal Perinatal Med 2010;3(2):135-139.

19. Rüegger C, Bucher HU, Mieth RA. Pulse oximetry in the newborn: is the left hand pre- or post-ductal? BMC Pediatr 2010;10:35.

20. Di Fiore JM, Bloom JN, Orge F, Schutt A, Schluchter M, Cheruvu $\mathrm{VK}$, et al. A higher incidence of intermittent hypoxemic episodes is associated with severe retinopathy of prematurity. J Pediatr 2010; 157(1):69-73 